\title{
Stochastic comparisons of distorted variability measures*
}

\author{
Miguel A. Sordo and Alfonso Suárez-Llorens \\ Departamento de Estadística e Investigación Operativa \\ Facultad de Ciencias Económicas y Empresariales \\ Universidad de Cádiz \\ 11002 Cádiz, Spain. \\ Tel.: +34-956-015403; fax: +34-956-016288 \\ E-mail addresses: mangel.sordo@uca.es, alfonso.suarez@uca.es
}

\begin{abstract}
In this paper, we consider the dispersive order and the excess wealth order to compare the variability of distorted distributions. We know from Sordo (2009a) that the excess wealth order can be characterized in terms of a class of variability measures associated to the tail conditional distribution which includes, as a particular measure, the tail variance. Given that the tail conditional distribution is a particular distorted distribution, a natural question is whether this result can be extended to include other classes of variability measures associated to general distorted distributions. As we show in this paper, the answer is yes, by focussing on distorted distributions associated to concave distortion functions. For distorted distributions associated to more general distortions, the characterizations are stated in terms of the stronger dispersive order.
\end{abstract}

MSC: IM30

Keywords: excess wealth order, dispersive order, distorted distributions, distortions, tail variance.

\section{Motivation}

Distorted distributions are of great interest because of their use in the Rank Dependent Expected Utility model (see Quiggin (1982), Yaari (1987) and Schmeidler (1989)). They were introduced in actuarial science by Denneberg $(1990)$ and Wang $(1995,1996)$ and have been applied

*This is a working paper. The final version has been published in Insurance: Mathematics and Economics (2011) Volume 49, Issue 1, Pages 11-17 
to a wide variety of insurance problems, most particularly to the determination of insurance premiums and risk measures (for further details see Goovaerts, Kaas and Laeven (2010) and the references therein).

In the literature one finds some papers connecting distorted distributions and stochastic orderings (for a general survey on this topic, see Shaked and Shanthikumar (2007); for applications of stochastic orders in actuarial science, see Müller and Stoyan (2002) and Denuit et al. (2005)). Two important contributions in this field are Chew, Karni and Safra (1987) and Wang and Young (1998) which show, in the framework of the distorted expectation hypothesis, that the increasing convex ordering (also called the stop-loss ordering) of two risks is equivalent to saying that one risk is preferred over the other by all decision-makers with concave distortion function. This result is important since every law invariant comonotonically additive coherent risk measures can be expressed as the expectation of a distorted distribution by a concave distortion function (Kusuoka, 2001).

Risk measures based on distorted expectations focus on the size of risks. However, although a measure of the size of the risk gives an important information about the riskiness of a loss distribution, very often it is not sufficient. This has been pointed out by a number of authors, who have suggested to complement these measures by using characteristics of variability to evaluate the uncertainty of the risk. An example of such characteristics is the tail variance, introduced by Furman and Landsman (2006) for measuring the variability of the risk along the right tail of its distribution (see also Furman and Zitikis (2008) and Landsman (2010)).

Sordo (2009a) generalizes the tail variance by considering a class of variability measures associated to the tail conditional distribution and characterizes this class in terms of another well-known stochastic order, namely the excess wealth order (also called the right spread order) of the risks. The motivation for our work comes from that characterization and the fact that the tail conditional distribution is nothing but a "particular" distorted distribution. Thus, the following question emerges: can we extend this result in order to characterize, by means of stochastic orders, the comparisons of risks in terms of other variability measures associated to "general" distorted distributions? The answer to this question is yes, as we show below, by considering certain general classes of variability measures associated to distorted distributions (from now on, we refer to these measures as distorted variability measures) and two stochastic orders well-known in actuarial context: the dispersive order (Bickel and Lehmann,1979) and the excess wealth order (Fernández-Ponce et al., 1998; Shaked and Shanthikumar, 1998). Our results parallel those given by Wang and Young (1998) in the framework of the distorted expecta- 
tion hypothesis, since we study under which circumstances the dispersive order and the excess wealth order are equivalent to saying that one risk is preferred over the other by all decision-makers who judge among them on the basis of distorted variability measures.

This paper is organized as follows. In Section 2, we introduce the classes of distorted variability measures and the stochastic orders that we consider later on. In Section 3 we characterize these stochastic orders in terms of classes of variance-type measures and in Section 4, we provide characterizations in terms of classes of Gini-type measures. In Section 5 , we obtain some conditions for stochastic equality of two ordered random variables in terms of some particular distorted variability measures. We illustrate the results on some parametric families of distributions in Section 6 and provide conclusions in Section 7 .

\section{Definitions}

In order to introduce the families of variability measures that will be considered in this paper, assume that we have an underlying risk described by a random variable $X$ defined on a probability space $(\Omega, B, P)$, where $\Omega$ is the sample space, $B$ is the $\sigma$-algebra and $P$ is the probability measure. Let $F$ be its distribution function, $F(x)=P[X \leq x]$, and let $\bar{F}=$ $1-F$ be its associated survival function. Denote by $F^{-1}$ the corresponding quantile function, defined by $F^{-1}(p)=\inf \{x: F(x) \geq p\}, 0 \leq p \leq 1$ and let $\bar{F}^{-1}(p)=F^{-1}(1-p)$ be the inverse of the survival function.

Consider the set $\Upsilon$ of continuous, non-decreasing and piecewise differentiable functions $h:[0,1] \Rightarrow[0,1]$, that satisfy $h(0)=0$ and $h(1)=1$

(such functions are called distortion functions). For each distortion $h \in \Upsilon$, the transformation of the survival function of $X$

$$
\bar{F}_{h}(x)=h[\bar{F}(x)]=h \circ \bar{F}(x)
$$

defines a new survival function associated to certain random variable $X_{h}$, which is the distorted random variable induced by $h$.

In insurance pricing and in financial risk management, a distortion typically represents a change in the probability measure. For instance, consider a risk $X$ with expectation

$$
E[X]=-\int_{-\infty}^{0}(1-\bar{F}(x)) d x+\int_{0}^{\infty} \bar{F}(x) d x
$$

(here and throughout this paper we assume that the integrals exist whenever they appear in the text). Under the distorted expectation hypothesis (see Section 2.6 in Denuit et al., 2005, for a review) it is assumed 
that each decision-maker has a distortion function $h$ and that he values $X$ as its distorted expectation $E_{h}[X]$, defined as

$$
E_{h}[X]=-\int_{-\infty}^{0}(1-h[\bar{F}(x)]) d x+\int_{0}^{\infty} h[\bar{F}(x)] d x .
$$

A concave distortion function gives more weight to higher risk events. For instance, Wang (1996) suggests to use (2) as a premium principle; for insurance premiums purposes, $E_{h}[X]$ must be at least equal to $E[X]$ and such is the case when $h$ is concave. Some important examples of distorted distribution with concave distortions are the following:

(a) If, given $p \in(0,1)$, we take $h(t)=\min \left\{\frac{t}{1-p}, 1\right\}$, then we have the tail conditional distribution (or conditional distribution at quantile $p$ )

$$
X_{h} \equiv\left[X \mid X>F^{-1}(p)\right] .
$$

(b) If $h(t)=1-(1-t)^{n}$, we have the maximum of $n$ independent and identically distributed under $F$ random variables

$$
X_{h} \equiv \max \left\{X_{1}, \ldots, X_{n}\right\} \text {. }
$$

(c) If $h(t)=\Phi\left(\Phi^{-1}(t)+\lambda\right), \lambda>0$, where $\Phi$ is the standard normal distribution function, then $X_{h} \equiv X_{W T}$ is the Wang transformed random variable (Wang, 2000). The expectation of this random variable is a well-known risk measure with many desirable properties. This distortion function was independently proposed by Goovaerts and Laeven (2008) under the guise of Esscher-Girsanov transform. They characterize a pricing mechanism involving this transform and also consider its dynamic extension (see also Labuschagne and Offwood, 2010).

A decision-maker who is concerned with the variability of extreme events (which typically represent events with a low frequency and high impact) may judge risks in terms of the variance associated to the above random variables or, more generally, in terms of some variability measure associated to $X_{h}$, where $h$ is a concave distortion function. Specifically, we focus on two classes of distorted variability measures. The first is the class of variance-type distorted variability measures, given by measures of the form

$$
I_{\varphi, h}(X)=E_{h}\left[\varphi\left(X-E_{h}[X]\right)\right]=-\int_{-\infty}^{\infty} \varphi\left(x-E_{h}[X]\right) d \bar{F}_{h}(x),
$$

where $\bar{F}_{h}$ is given by (1) and $\varphi$ is a convex function defined on $[0,1]$. The convexity of $\varphi$ ensures that (3) generalizes the variance of $X$, which 
is given by the choice $h(t)=t$ and $\varphi(t)=t^{2}$. Note that a functional of the form (3) represents a measure of the distance between $X$ and $E_{h}[X]$ when the deviations are evaluated under the distorted probability induced by $h$. Thus, for example, if we combine the $h^{\prime} \mathrm{s}$ in the above examples with $\varphi(t)=t^{2}$, we obtain, respectively, the tail variance,

$$
\operatorname{Var}\left[X \mid X>F^{-1}(p)\right]
$$

the variance of the maximum of $n$ independent copies of $X$,

$$
\operatorname{Var}\left[\max \left\{X_{1}, \ldots, X_{n}\right\}\right]
$$

and the variance associated to the Wang transformed random variable,

$$
\operatorname{Var}\left[X_{W T}\right] .
$$

The choice $\varphi(t)=(\max \{0, t\})^{2}$ leads to the positive semivariance and, in general, different $\varphi^{\prime}$ s and $h$ 's produce different variability measures associated to different distorted distributions. Note that we can also take a convex distortion $h$ in (3); in such a case, we are focusing on the variability of the left-tail of the risk. For example, the choice $\varphi(t)=t^{2}$ and $h(t)=t^{n}$ produces the variance of the minimum of $n$ independent copies of $X$,

$$
\operatorname{Var}\left[\min \left\{X_{1}, \ldots, X_{n}\right\}\right] .
$$

The second class of measures considered in this paper is a family of Ginitype distorted variability measures, given by functionals of the form

$$
G_{\alpha, h}(X)=\int_{0}^{1}\left(\bar{F}_{h}^{-1}(t)-E_{h}[X]\right) d \alpha(t)
$$

where $\alpha$ is a concave weight function defined on $[0,1]$. The concavity of $\alpha$ ensures that (8) generalizes the Gini's mean difference of $X$ (denoted by $G M D(X)$ ), which is given by the choice $h(t)=t$ and $\alpha(t)=2\left[1-(1-t)^{2}\right]$. If we combine this $\alpha$ with the $h^{\prime} \mathrm{s}$ in the above examples, we obtain the Gini's mean difference associated, respectively, to the conditional distribution at quantile $p$, the maximum of $n$ independent copies of $X$ and the Wang transformed random variable.

In this paper, we are interested in how a collection of decision-makers order risks by considering orders based on the conditions

$$
I_{\varphi, h}(X) \leq I_{\varphi, h}(Y) \text { for all } h \in \Psi, \text { for all convex } \varphi
$$

and

$$
G_{\alpha, h}(X) \leq G_{\alpha, h}(Y) \text { for all } h \in \Lambda, \text { for all concave } \alpha
$$


where $\Psi$ and $\Lambda$ are certain classes of distortion functions. Judgements based on this type of comparisons do not depend on subjective distortion functions. A similar problem, involving different stochastic orders and measures, has recently been studied by Shaked, Sordo and SuárezLlorens (2010).

Now we recall the definition of the stochastic orders that we consider in this paper.

Definition 1 Let $X$ and $Y$ be two random variables with respective distribution functions $F$ and $G$. Then,

(i) $X$ is said to be smaller than $Y$ in the dispersive order (denoted by $\left.X \leq_{\text {disp }} Y\right)$ if $F^{-1}(p)-F^{-1}(q) \leq G^{-1}(p)-G^{-1}(q)$, for all $0<q<p<1$. (ii) $X$ is said to be smaller than $Y$ in the excess wealth order (denoted by $\left.X \leq_{e w} Y\right)$ if $E\left[\left(X-F^{-1}(p)\right)^{+}\right] \leq E\left[\left(Y-G^{-1}(p)\right)^{+}\right]$for all $p \in(0,1)$, where $x^{+}=\max \{x, 0\}$.

The dispersive order and the excess wealth order have been extensively studied in the literature and have been applied before to insurance problems by Denuit and Vermandele (1999), Chateauneuf et al. (2004), $\mathrm{Hu}$, Chen and Yao (2006) and Sordo (2008, 2009a, 2009b). The excess wealth order is also termed as the right-spread order (Fernández-Ponce, Kochar and Muñoz-Pérez, 1998) and the shortfall order (Denuit, Goderniaux and Sacillet, 2007). Many properties of these orders can be found in Chapter 3 of Shaked and Shanthikumar (2007). Since $X \leq_{\text {disp }} Y$ implies $X \leq_{e w} Y$ and the reverse is not true, the excess wealth order is useful to compare the variability among random variables when the dispersive order does not hold.

\section{Characterizations in terms of variance-type dis- torted variability measures}

In this section, we consider orders of the form (9) for the following classes of distorted variability measures

$$
\begin{gathered}
\Psi_{1}=\left\{I_{\varphi, h} \text { of the form }(3) \text { with } \varphi \text { convex }\right\} \\
\Psi_{2}=\left\{I_{\varphi, h} \in \Psi_{1} \text { with } h \text { concave }\right\}
\end{gathered}
$$

Some members of $\Psi_{i}, i=1,2$, are (4), (5) and (6). The functional (7) is an example of member of $\Psi_{1}$ not belonging to $\Psi_{2}$. It is clear, from these examples and the discussion in the previous section, that members of $\Psi_{1}$ take into account the variability throughout the whole distribution, whereas members of $\Psi_{2}$ focus on the right tail variability. 
The main results are obtained as a consequence of the theory of majorization (see Hardy, Littlewood and Pólya (1929) and Chong (1974)). Denote by $M(\Omega, \mu)$ the set of all extended real-valued measurable functions on a measure space $(\Omega, \Lambda, \mu)$. The decreasing rearrangement of $f \in M(\Omega, \mu)$ is defined by

$$
f^{*}(t)=\inf \left\{s \in \mathbf{R}: D_{f}(s) \leq t\right\}, t \in[0, \mu(\Omega)]
$$

where

$$
D_{f}(s)=\mu(\{x: f(x)>s\}),
$$

for each $s \in[-\infty, \infty]$. The following result is taken from Chong (1974).

Theorem 2 Suppose $(\Omega, \Lambda, \mu)$ and $\left(\Omega^{\prime}, \Lambda^{\prime}, \mu^{\prime}\right)$ are measure spaces such that $\mu(\Omega)=\mu^{\prime}\left(\Omega^{\prime}\right)=a<\infty$ and denote by $m$ the Lebesgue measure on $\mathbf{R}$. If $f \in L^{1}(\Omega, \mu)$ and $g \in L^{1}\left(\Omega^{\prime}, \mu^{\prime}\right)$, the following conditions are equivalent.

(a) $\int_{0}^{t} f^{*} d m \leq \int_{0}^{t} g^{*} d m$ for all $t \in[0, a)$ and $\int_{0}^{a} f^{*} d m=\int_{0}^{a} g^{*} d m$.

(b) $\int_{\Omega} \varphi(f) d \mu \leq \int_{\Omega^{\prime}} \varphi(g) d \mu^{\prime}$ for all convex functions $\varphi: \mathbf{R} \longrightarrow \mathbf{R}$.

First we have to prove the following result.

Theorem 3 Let $X$ and $Y$ be two random variables with distribution functions $F$ and $G$, respectively and let $h \in \Upsilon$. For each $u \in[0,1]$ denote

$$
L_{h}^{X}(u)=\int_{0}^{u}\left(\bar{F}_{h}^{-1}(t)-E_{h}[X]\right) d t
$$

and

$$
L_{h}^{Y}(u)=\int_{0}^{u}\left(\bar{G}_{h}^{-1}(t)-E_{h}[Y]\right) d t .
$$

Then, $I_{\varphi, h}(X) \leq I_{\varphi, h}(Y)$ for all convex $\varphi$ if and only if

$$
L_{h}^{X}(u) \leq L_{h}^{Y}(u) \text { for all } u \in(0,1) .
$$

Proof. Let $\left(\Omega, B, P_{X}^{h}\right)$ and $\left(\Omega^{\prime}, B^{\prime}, P_{Y}^{h}\right)$ be the probability spaces on which $X_{h}$ and $Y_{h}$, respectively, are defined. Define $f(\omega)=X(\omega)-E_{h}[X]$ for all $\omega \in \Omega$ and $g(\omega)=Y(\omega)-E_{h}[Y]$ for all $\omega \in \Omega^{\prime}$. The decreasing rearrangements of $f$ and $g$ are given, respectively, by

$$
f^{*}(t)=\bar{F}^{-1}\left(h^{-1}(t)\right)-E_{h}[X]
$$

and

$$
g^{*}(t)=\bar{G}^{-1}\left(h^{-1}(t)\right)-E_{h}[Y],
$$


for all $t \in[0,1]$. Let us apply Theorem 2 to these probability spaces. First, note that Theorem 2(b) reads as $I_{\varphi, h}(X) \leq I_{\varphi, h}(Y)$ for all $\varphi$ convex. In order to verify condition (a), note that the equality

$$
\int_{0}^{1}\left(\bar{F}^{-1}\left(h^{-1}(t)\right)-E_{h}[X]\right) d x=\int_{0}^{1}\left(\bar{G}^{-1}\left(h^{-1}(t)\right)-E_{h}[Y]\right) d x
$$

follows as a consequence of the following representation ${ }^{1}$ for (2) (see Jones and Zitikis, 2003):

$$
E_{h}[X]=\int_{0}^{1} \bar{F}^{-1}(t) d h(t)
$$

The rest of the condition (a) in Theorem 2 reads as

$$
\begin{gathered}
\int_{0}^{u}\left(\bar{F}^{-1}\left(h^{-1}(t)\right)-E_{h}[X]\right) d t \leq \\
\leq \int_{0}^{u}\left(\bar{G}^{-1}\left(h^{-1}(t)\right)-E_{h}[Y]\right) d t, \text { for all } u \in(0,1),
\end{gathered}
$$

which is equivalent to (13) and this ends the proof.

The following result characterizes the comparisons of functionals of the form (3) by means of the dispersive order and the excess wealth order. Part (b) of this theorem extends Theorem 4 of Sordo (2009a) in the following sense: whereas in Sordo (2009a) the excess wealth order is shown to be monotone with respect to a class of variability measures associated to the conditional distribution at quantile $p$ (which is a particular distorted distribution), here this order is shown to be monotone with respect to general distorted distributions, whenever the corresponding distortions are concave. Recall that $\Psi_{1}$ and $\Psi_{2}$ are defined in (11).

Theorem 4 Let $X$ and $Y$ be two random variables with distribution functions $F$ and $G$, respectively. Then,

(a) $X \leq_{\text {disp }} Y$ if, and only if, $I_{\varphi, h}(X) \leq I_{\varphi, h}(Y)$ for all $I_{\varphi, h} \in \Psi_{1}$.

(b) $X \leq_{e w} Y$ if, and only if, $I_{\varphi, h}(X) \leq I_{\varphi, h}(Y)$ for all $I_{\varphi, h} \in \Psi_{2}$.

Proof. (a) Assume that $X \leq_{\text {disp }} Y$. Then, from Theorem 7(i) of Sordo (2008) it follows that (13) holds for all $h \in \Upsilon$ and from Theorem 3 it follows that $I_{\varphi, h}(X) \leq I_{\varphi, h}(Y)$ for all $I_{\varphi, h} \in \Psi_{1}$. In order to prove the

\footnotetext{
${ }^{1}$ Recall that $\Upsilon$ is the set of continuous and piecewise differentiable distortion functions, therefore this representation is valid.
} 
converse, assume that $I_{\varphi, h}(X) \leq I_{\varphi, h}(Y)$ for all $I_{\varphi, h} \in \Psi_{1}$. In particular, if we take $h=h_{p, q}$, with $0 \leq p<q \leq 1$, defined by

$$
h_{p, q}(t)=\left\{\begin{array}{c}
0 \text { if } 0 \leq t<p \\
\frac{t-p}{q-p} \text { if } p \leq t \leq q \\
1 \text { if } q<t \leq 1
\end{array},\right.
$$

we have

$$
I_{\varphi, h_{p, q}}(X) \leq I_{\varphi, h_{p, q}}(Y) \text { for all } 0<p<q<1 \text {, for all convex } \varphi .
$$

Using the representation (16), we see that

$$
E_{h_{p, q}}[X]=\frac{\int_{p}^{q} \bar{F}^{-1}(s) d s}{q-p} .
$$

Combining (17) and Theorem 3 it follows that

$$
L_{h_{p, q}}^{X}(u) \leq L_{h_{p, q}}^{Y}(u) \text { for all } u \in(0,1), \text { for all } 0<p<q<1,
$$

or, equivalently,

$$
\begin{aligned}
& \int_{0}^{u}\left(\bar{F}^{-1}(t)-\frac{\int_{p}^{q} \bar{F}^{-1}(s) d s}{q-p}\right) d h_{p, q}(t) \leq \\
& \leq \int_{0}^{u}\left(\bar{G}^{-1}(t)-\frac{\int_{p}^{q} \bar{G}^{-1}(s) d s}{q-p}\right) d h_{p, q}(t)
\end{aligned}
$$

for all $u \in(0,1)$, for all $0<p<q<1$.

The above expression is the same as

$$
\begin{gathered}
\int_{p}^{u}\left(\bar{F}^{-1}(t)-\frac{\int_{p}^{q} \bar{F}^{-1}(s) d s}{q-p}\right) d t \leq \int_{p}^{u}\left(\bar{G}^{-1}(t)-\frac{\int_{p}^{q} \bar{G}^{-1}(s) d s}{q-p}\right) d t \\
\text { for all } 0<p<u<q<1 .
\end{gathered}
$$

Equivalently, we can write

$$
\begin{gathered}
\frac{1}{u-p} \int_{p}^{u}\left(\bar{F}^{-1}(t)-\bar{G}^{-1}(t)\right) d t \leq \frac{1}{q-p} \int_{p}^{q}\left(\bar{F}^{-1}(s)-\bar{G}^{-1}(s)\right) d u \\
\text { for all } 0<p<u<q<1
\end{gathered}
$$


which means that, for each $p \in(0,1)$,

$$
\frac{1}{x-p} \int_{p}^{x}\left(\bar{F}^{-1}(t)-\bar{G}^{-1}(t)\right) d t
$$

is an increasing function of $x$ on $(0,1)$. By differentiation, it is seen that (18) holds if, and only if,

$$
\int_{p}^{x}\left(\bar{F}^{-1}(x)-\bar{F}^{-1}(t)\right) d t \geq \int_{p}^{x}\left(\bar{G}^{-1}(x)-\bar{G}^{-1}(t)\right) d t
$$

for all $x \in(p, 1)$. Now, by contradiction, suppose that $X \not \leq_{d i p} Y$. Recalling that $X \leq_{\text {disp }} Y$ if and only if $\bar{F}^{-1}(t)-\bar{G}^{-1}(t)$ is non-decreasing (see (3.B.8) in Shaked and Shanthikumar, 2007), the contradiction argument implies that there exists an interval $\left(p_{0}, x_{0}\right) \subset(0,1)$ such that

$$
\bar{F}^{-1}(t)-\bar{G}^{-1}(t)>\bar{F}^{-1}\left(x_{0}\right)-\bar{G}^{-1}\left(x_{0}\right) \text { for all } t \in\left(p_{0}, x_{0}\right) .
$$

This implies that

$$
\int_{p_{0}}^{x_{0}}\left(\bar{F}^{-1}\left(x_{0}\right)-\bar{F}^{-1}(t)\right) d t<\int_{p_{0}}^{x_{0}}\left(\bar{G}^{-1}\left(x_{0}\right)-\bar{G}^{-1}(t)\right) d t
$$

a contradict with (19). This proves that $X \leq_{\text {disp }} Y$.

(b) From Theorem 7(ii) of Sordo (2008) it follows that $X \leq_{e w} Y$ if, and only if, (13) holds for all concave $h$ and, from Theorem 3 , this is equivalent to say that $I_{\varphi, h}(X) \leq I_{\varphi, h}(Y)$ for all $I_{\varphi, h} \in \Psi_{2}$.

\section{Characterizations in terms of Gini-type distorted variability measures}

In this section, we consider orders of the form (10) for the following classes of distorted variability measures

$$
\Lambda_{1}=\left\{G_{\alpha, h} \text { of the form (8) with } \alpha \text { concave }\right\}
$$

and

$$
\Lambda_{2}=\left\{G_{\alpha, h} \in \Theta_{1} \text { with } h \text { concave }\right\} .
$$

Note that $\Lambda_{1}$ includes the Gini's mean difference associated to any distorted distribution, that is, it takes into account the variability throughout the whole distribution. The class $\Lambda_{2}$ contains measures associated to distorted distribution whit $h$ concave, that is, it focusses on the variability of the tail right of distributions. 
Theorem 5 Let $X$ and $Y$ be two random variables with distribution functions $F$ and $G$, respectively. Then,

(a) $X \leq_{\text {disp }} Y$ if, and only if, $G_{\alpha, h}(X) \leq G_{\alpha, h}(Y)$ for all $G_{\alpha, h} \in \Lambda_{1}$.

(b) $X \leq_{e w} Y$ if, and only if, $G_{\alpha, h}(X) \leq G_{\alpha, h}(Y)$ for all $G_{\alpha, h} \in \Lambda_{2}$.

Proof. First, we provide an alternative representation for every $G_{\alpha, h} \in$ $\Lambda_{1}$. From the concavity of $\alpha$ on $[0,1]$, there exists a non-increasing function $\omega$ such that

$$
\alpha(p)-\alpha(0)=\int_{0}^{p} \omega(t) d t, \quad p \in[0,1)
$$

(see Chow and Teicher (1997), page 428). Now, by using (12) and (20) we can write

$$
G_{\alpha, h}(X)=\int_{0}^{1}\left(\bar{F}_{h}^{-1}(t)-E_{h}[X]\right) d \alpha(t)=\int_{0}^{1} \omega(t) d L_{h}^{X}(t) .
$$

Integration by parts and the fact that $L_{h}^{X}(0)=L_{h}^{X}(1)=0$ imply that

$$
G_{\alpha, h}(X)=-\int_{0}^{1} L_{h}^{X}(t) d \omega(t) .
$$

In order to prove part (a), assume that $X \leq_{d i s p} Y$. Combining theorems 3 and 4 , we see that this is equivalent to say that

$$
L_{h}^{X}(u) \leq L_{h}^{Y}(u) \text { for all } u \in(0,1), \text { for all distortion } h .
$$

Since $d \omega(t) \leq 0$ in (21), it follows from (21) and (22) that $G_{\alpha, h}(X) \leq$ $G_{\alpha, h}(Y)$ for all $G_{\alpha, h} \in \Lambda_{1}$. Conversely, assume that $G_{\alpha, h}(X) \leq G_{\alpha, h}(Y)$ for all $G_{\alpha, h} \in \Lambda_{1}$. In particular, by taking the concave function $\alpha_{u}(t)=$ $\min \{t, u\}, u \in(0,1)$, we see that

$$
G_{\alpha_{u}, h}(X)=\int_{0}^{u}\left(\bar{F}_{h}^{-1}(t)-E_{h}[X]\right) d t=L_{h}^{X}(u)
$$

is a member of $\Lambda_{1}$ for each $u \in(0,1)$. Thus,

$$
G_{\alpha_{u}, h}(X) \leq G_{\alpha_{u}, h}(Y), \text { for all } u \in(0,1)
$$

holds, which is the same as (22), that is, $X \leq_{\text {disp }} Y$.

The proof of part (b) follows the same lines as the proof of part (a) by using that $X \leq_{e w} Y$ is equivalent to say that

$$
L_{h}^{X}(u) \leq L_{h}^{Y}(u) \text { for all } u \in(0,1) \text {, for all concave } h
$$

(which follows by combining theorems 3 and 4). 


\section{Conditions for stochastic equality of distributions}

It is well-known that two random variables with the same variance are not ordered in the excess wealth order unless they are stochastically equivalent up to a location parameter. In this section, we provide some new conditions for stochastic equality of two ordered random variables under the dispersive order and the excess wealth order in terms of some special variability measures associated to certain distorted random variables. For example, it will be proven that two random variables whose corresponding Wang transformed have the same variance, can not be ordered in the excess wealth order unless they have, up to a location parameter, the same distribution.

The first result in this section follows from the following result of Chong (1974).

Theorem 6 Suppose $(\Omega, \Lambda, \mu)$ and $\left(\Omega^{\prime}, \Lambda^{\prime}, \mu^{\prime}\right)$ are measure spaces such that $\mu(\Omega)=\mu^{\prime}\left(\Omega^{\prime}\right)=a<\infty$ and denote by $m$ the Lebesgue measure on R. Assume that $f \in L^{1}(\Omega, \mu)$ and $g \in L^{1}\left(\Omega^{\prime}, \mu^{\prime}\right)$. If

$$
\int_{0}^{t} f^{*} d m \leq \int_{0}^{t} g^{*} d m \text { for all } t \in[0, a) \text { and } \int_{0}^{a} f^{*} d m=\int_{0}^{a} g^{*} d m
$$

and

$$
\int_{\Omega} \varphi(f) d \mu=\int_{\Omega^{\prime}} \varphi(g) d \mu^{\prime}
$$

for some strictly convex function $\varphi$, then

$$
\mu \circ f^{-1}=\mu^{\prime} \circ g^{-1}
$$

Corollary 7 Let $X$ and $Y$ be two random variables with distribution functions $F$ and $G$, respectively. Let $h$ be a distortion function. If

$$
L_{h}^{X}(u) \leq L_{h}^{Y}(u) \text { for all } u \in(0,1)
$$

and

$$
I_{\varphi, h}(X)=I_{\varphi, h}(Y)
$$

for some $I_{\varphi, h}$ with strictly convex $\varphi$, then

$$
\bar{F}_{h}\left(t+E_{h}[X]\right)=\bar{G}_{h}\left(t+E_{h}[Y]\right) \text { for all } t .
$$

Proof. Let $h$ be a distortion function and, as in the proof of Theorem 3 , let $\left(\Omega, B, P_{X}^{h}\right)$ and $\left(\Omega^{\prime}, B^{\prime}, P_{Y}^{h}\right)$ be the probability spaces on which $X_{h}$ and $Y_{h}$, respectively, are defined. Consider again the functions $f(\omega)=$ $X(\omega)-E_{h}[X]$ for all $\omega \in \Omega$ and $g(\omega)=Y(\omega)-E_{h}[Y]$ for all $\omega \in \Omega^{\prime}$. 
The decreasing rearrangements of $f$ and $g$ are given, respectively, by (14) and (15). Now the result follows by applying Theorem 6 to these probability spaces. As in the proof of Theorem 3, condition (25) reads as (28) and condition (26) reads as (29). Finally, it is easy to see that (27) is the same as (30).

Corollary 8 Let $X$ and $Y$ be two random variables with distribution functions $F$ and $G$, respectively.

(a) If $X \leq_{\text {disp }} Y$ and $I_{\varphi, h}(X)=I_{\varphi, h}(Y)$ for some $I_{\varphi, h}$ with strictly convex $\varphi$ and strictly increasing $h$, then $X$ and $Y$ have the same distribution, up to a location parameter.

(b) If $X \leq_{e w} Y$ and $I_{\varphi, h}(X)=I_{\varphi, h}(Y)$ for some $I_{\varphi, h}$ with strictly convex $\varphi$ and strictly increasing and concave $h$, then $X$ and $Y$ have the same distribution, up to a location parameter.

Proof. (a) If $X \leq_{\text {disp }} Y$, from (22) it follows that (28) holds for all distortion function $h$. It follows from Corollary 7 that

$$
h\left(\bar{F}\left(t+E_{h}[X]\right)\right)=h\left(\bar{G}\left(t+E_{h}[Y]\right)\right) \text { for all } t
$$

which, by the strictly increasing of $h$, is equivalent to say that

$$
X-E_{h}[X] \equiv_{s t} Y-E_{h}[Y]
$$

This means that $X$ and $Y$ have the same distribution, up to a location parameter.

(b) If $X \leq_{\text {ew }} Y$, from (24) it follows that (28) holds for all concave distortion function $h$ and the proof follows the same lines as in part (a).

Example 9 Let $X$ and $Y$ be two random variables with distribution functions $F$ and $G$, respectively. Let $X_{1}, \ldots, X_{n}$ be independent copies of $X$ and let $Y_{1}, \ldots, Y_{n}$ be independent copies of $Y$.

(a) If $X \leq_{\text {disp }} Y$ and $\operatorname{Var}\left[\min \left(X_{1}, \ldots, X_{n}\right)\right]=\operatorname{Var}\left[\min \left(Y_{1}, \ldots, Y_{n}\right)\right]$, then $X$ and $Y$ have the same distribution, up to a location parameter.

(b) If $X \leq_{e w} Y$ and $\operatorname{Var}\left[\max \left(X_{1}, \ldots, X_{n}\right)\right]=\operatorname{Var}\left[\max \left(Y_{1}, \ldots, Y_{n}\right)\right]$, then $X$ and $Y$ have the same distribution, up to a location parameter.

(c) If $X \leq_{e w} Y$ and $\operatorname{Var}\left[X_{W T}\right]=\operatorname{Var}\left[Y_{W T}\right]$, where $X_{W T}$ and $Y_{W T}$ are the Wang transformed random variables associated to $X$ and $Y$, respectively, then $X$ and $Y$ have the same distribution, up to a location parameter.

We also have the following result. 
Theorem 10 Let $X$ and $Y$ be two random variables with distribution functions $F$ and $G$, respectively.

(a) If $X \leq_{\text {disp }} Y$ and $G_{\alpha, h}(X)=G_{\alpha, h}(Y)$ for some $G_{\alpha, h}$ with strictly convex $\alpha$ and strictly increasing $h$, then $X$ and $Y$ have the same distribution, up to a location parameter.

(b) If $X \leq_{e w} Y$ and $G_{\alpha, h}(X)=G_{\alpha, h}(Y)$ for some $G_{\alpha, h}$ with strictly convex $\alpha$ and strictly increasing and concave $h$, then $X$ and $Y$ have the same distribution, up to a location parameter.

Proof. (a) If $X \leq_{\text {disp }} Y$, then (22) holds. Assume that $G_{\alpha, h}(X)=$ $G_{\alpha, h}(Y)$ for some $G_{\alpha, h}$ with strictly convex $\alpha$ and strictly increasing $h$. By using (21), this means that there exists a strictly decreasing function $\omega$ such that

$$
\int_{0}^{1} L_{h}^{X}(t) d \omega(t)=\int_{0}^{1} L_{h}^{Y}(t) d \omega(t),
$$

or, equivalently,

$$
\int_{0}^{1}\left[L_{h}^{X}(t)-L_{h}^{Y}(t)\right] d \omega(t)=0 .
$$

From (22) and the strict monotony of $\omega$, it follows that $L_{h}^{X}(t)=L_{h}^{Y}(t)$ for all $t$ and, by differentiation, we get

$$
\bar{F}_{h}^{-1}(t)-E_{h}[X]=\bar{G}_{h}^{-1}(t)-E_{h}[Y] \text {, for all } t .
$$

Equivalently, we can write

$$
\bar{F}^{-1}\left(h^{-1}(t)\right)-E_{h}[X]=\bar{G}^{-1}\left(h^{-1}(t)\right)-E_{h}[Y] \text { for all } t .
$$

Since $h$ is strictly increasing, this is the same as

$$
\bar{F}^{-1}(u)=\bar{G}^{-1}(u)+\left(E_{h}[X]-E_{h}[Y]\right)
$$

which means that $X$ and $Y$ have the same distribution, up to a location parameter.

(b) If $X \leq_{e w} Y$, then (24) holds and the proof follows the same lines as in part (a).

Example 11 Let $X$ and $Y$ be two random variables with distribution functions $F$ and $G$, respectively. Let $X_{1}, \ldots, X_{n}$ be independent copies of $X$, let $Y_{1}, \ldots, Y_{n}$ be independent copies of $Y$ and let $G M D(\cdot)$ denote the Gini's mean difference.

(a) If $X \leq_{d i s p} Y$ and $G M D\left[\min \left(X_{1}, \ldots, X_{n}\right)\right]=G M D\left[\min \left(Y_{1}, \ldots, Y_{n}\right)\right]$, 
then $X$ and $Y$ have the same distribution, up to a location parameter. (b) If $X \leq_{e w} Y$ and $G M D\left[\max \left(X_{1}, \ldots, X_{n}\right)\right]=G M D\left[\max \left(Y_{1}, \ldots, Y_{n}\right)\right]$, then $X$ and $Y$ have the same distribution, up to a location parameter. (c) If $X \leq_{e w} Y$ and $G M D\left[X_{W T}\right]=G M D\left[Y_{W T}\right]$, where $X_{W T}$ and $Y_{W T}$ are the Wang transformed random variables associated to $X$ and $Y$, respectively, then $X$ and $Y$ have the same distribution, up to a location parameter.

\section{Application to the comparison of parametric fam- ilies of distributions}

Explicit expressions for some distorted variability measures have been derived in particular cases. For example, it is well-known (Wang, 2000) that if $X$ has a Normal distribution, $X \sim N\left(\mu_{1}, \sigma_{1}\right)$, then the Wang transformed random variable $X_{W T}$ also follows a Normal distribution with $\mu_{1}^{\prime}=\mu_{1}+\lambda \sigma_{1}$ and $\sigma_{1}^{\prime}=\sigma_{1}$, therefore

$$
\operatorname{Var}\left[X_{W T}\right]=\operatorname{Var}[X]=\sigma_{1}
$$

Another distorted variability measure is the tail variance; Furman and Landsman (2006) show that when $X \sim N\left(\mu_{1}, \sigma_{1}\right)$, the tail variance equals

$$
\operatorname{Var}\left[X \mid X>F^{-1}(p)\right]=\sigma_{1}\left[1+\frac{\varphi\left(z_{p}\right)}{1-\Phi\left(z_{p}\right)}\left(z_{q}-\frac{\varphi\left(z_{p}\right)}{1-\Phi\left(z_{p}\right)}\right)\right]
$$

where $\varphi$ and $\Phi$ are the density function and the distribution function, respectively, of $N(0,1)$ and $z_{p}$ is the corresponding $p$-quantile, $0<p<1$. If $Y$ is another random variable such that $Y \sim N\left(\mu_{2}, \sigma_{2}\right)$, with $\sigma_{1} \leq \sigma_{2}$, the inequalities

$$
\operatorname{Var}\left[X_{W T}\right] \leq \operatorname{Var}\left[Y_{W T}\right]
$$

and

$$
\operatorname{Var}\left[X \mid X>F^{-1}(p)\right] \leq \operatorname{Var}\left[Y \mid Y>F^{-1}(p)\right], p \in(0,1)
$$

follow easily from (31) and (32). In many cases, however, direct comparisons are not possible since analytic expressions for the variability measures are not available. In those situations, the results obtained in sections 2 and 3 can prove to be very useful. Since several well-known parametric families of distributions are ordered in the dispersive order according to the value of their parameters, we can, for these families, compare risks in terms of these measures without needing their explicit expressions. We give an example to illustrate this application. 
Example 12 Let $t_{n}$ be at student distribution with $n$ degrees of freedom and density function given by

$$
f(x)=\frac{\Gamma\left(\frac{n+1}{2}\right)}{\sqrt{n \pi} \Gamma\left(\frac{n}{2}\right)}\left(1+\frac{t^{2}}{n}\right)^{-\left(\frac{n+1}{2}\right)},
$$

where $\Gamma(\cdot)$ denotes the complete gamma function. Consider two random variables $X(n)$ and $X(m)$ such that $X(n) \sim t_{n}$ and $X(m) \sim t_{m}$, with $n<m$. It is well-known (Arias-Nicolás et al, 2005), that $X(m) \leq_{\text {disp }}$ $X(n)$ and, from Theorem 4, it follows that

$$
\begin{gathered}
\operatorname{Var}\left[X(m) \mid X(m)>F_{X(m)}^{-1}(p)\right] \leq \operatorname{Var}\left[X(n) \mid X(n)>F_{X(n)}^{-1}(p)\right] \\
\text { for all } p \in(0,1), \\
\operatorname{Var}\left[[X(m)]_{W T}\right] \leq \operatorname{Var}\left[[X(n)]_{W T}\right]
\end{gathered}
$$

and, in general,

$$
I_{\varphi, h}(X(m)) \leq I_{\varphi, h}(X(n)) \text { for all distortion } h \text { and } \varphi \text { convex. }
$$

Similarly, from Theorem 5, it follows that

$$
\begin{gathered}
G M D\left[X(m) \mid X(m)>F_{X(m)}^{-1}(p)\right] \leq G M D\left[X(n) \mid X(n)>F_{X(n)}^{-1}(p)\right] \\
\text { for all } p \in(0,1),
\end{gathered}
$$

and, in general,

$$
G_{\alpha, h}(X(m)) \leq G_{\alpha, h}(X(n)) \text { for all distortion } h \text { and } \varphi \text { concave. }
$$

The intuitive meaning of this is that if we distort two $t$ Student distributions, one being more variable than the other, the corresponding distorted distributions are ordered, in terms of variability, in the same direction.

There are many other families of parametric distributions ordered in the dispersive ordering (and, therefore, in the excess wealth order) according to the value of their parameters. It is well-known, for example, that the normal family is ordered attending to the value of the variance, the gamma family attending to both the shape and scale parameters -which in turns includes both the exponential and chi-squared families-, the uniform family attending to the length of the interval, the Pareto family attending to both the minimum value and the Pareto index parameter. These and other examples of dispersive-ordered families of distributions can be found in Saunders and Moran (1978), Lewis and Thomson (1981), Shaked (1982), Hickey (1986) and Rojo and He (1991), among others. 


\section{Conclusions}

In this paper, we have shown that the dispersive order and the excess wealth order are monotone with respect to large classes of variability measures associated with distorted distributions. We have considered two types of variability measures, variance-type measures and Gini-type measures, and we have proven that these measures are consistent with respect to the dispersive order. Moreover, the variability measures associated to distorted distributions with concave distortion functions are also consistent with respect to the excess wealth order. Finally, we have provided some sufficient conditions, in terms of these orders and measures, for stochastic equality (up to a location parameter) of the underlying distributions.

\section{Aknowledgement}

We are grateful to the referee for his helpful comments and remarks, which resulted in a improved version of this paper. This research was

supported by Ministerio de Ciencia e Innovación (grant MTM2009-08326) and Consejería de Economía Innovación y Ciencia (grant P09-SEJ-4739).

\section{References}

Arias-Nicolás, J.P., Fernández-Ponce, J. M., Luque-Calvo, P., SuárezLlorens, A., 2005. The multivariate dispersion order and the notion of copula applied to the multivariate t-distribution. Probability in the Engineering and Informational Science. 19, 363-375.

Bickel, P.J., Lehmann, E.L., 1979. Descriptive statistics for nonparametric models IV. Spread. In: Jureckova (Ed.), Contributions to Statistics. Reidel, Dordrecht.

Chateauneuf, A., Cohen, M., Meilijson., I., 2004. Four notions of meanpreserving increase in risk, risk attitudes and applications to the rank-dependent expected utility model. Journal of Mathematical Economics 40, 547-571.

Chew Soo H., Karni, E., Safra, S., 1987. Risk aversion in the theory of expected utility with rank dependent probabilities. Journal of Economic Theory 42, 370-381.

Chong, K.M., 1974. Some extensions of a theorem of Hardy, Littlewood and Pólya and their applications. Canad. J. Math. 26, 1321-1340. 
Chow, Y.S., Teicher, H. 1997. Probability Theory. Independence, interchangeability, martingales. Third Edition. Springer.

Denneberg, D., 1990. Premium calculation: Why standard deviation should be replaced by absolute deviation. ASTIN Bulletin 20, 181190.

Denuit, M., Dhaene, J., Goovaerts, M.J., Kaas, R., 2005. Actuarial Theory for Dependent Risks. John Wiley\&Sons.

Denuit, M., Goderniaux, A-C., Scaillet, O., 2007. A KolmogorovSmirnov type test for shortfall dominance against parametric alternatives. Technometrics 49, 88-98.

Denuit, M., Vermandele, C., 1999. Lorenz and excess wealth orders, with applications in reinsurance theory. Scandinavian Actuarial Journal 2, 170-185.

Fernández-Ponce, J.M., Kochar, S.C., Muñoz-Perez, J., 1998. Partial orderings of distributions based on right-spread functions. Journal of Applied Probability 35, 221-228.

Furman, E., Landsman, Z., 2006. Tail Variance Premium with Applications for Elliptical Portfolio of Risks. ASTIN Bulletin 36, 433-462.

Furman, E., Zitikis, R., 2008. Weighted premium calculation principles. Insurance: Mathematics and Economics 42, 459-465.

Goovaerts, M.J., Laeven, R.J.A., 2008. Actuarial risk measures for financial derivative pricing. Insurance: Matematics and Economics $42,540-547$.

Goovaerts, M.J., Kaas, R., Laeven, R.J.A., 2010. Decision principles derived from risk measures. Insurance: Mathematics and Economics 47, 294-302

Hardy, G.H., Littlewood, J.E., Pólya, G., 1929. Some simple inequalities satisfied by convex functions. Mess. Math. 58, 145-152.

Hickey, R. J., 1986. Concepts of dispersion in distributions: a comparative note. J. Appl. Prob. 23, 914-921.

Hu, T., Chen, J., Yao, J., 2006. Preservation of the location independent risk order under convolution. Insurance: Mathematics and Economics 38, 406-412. 
Kusuoka, S., 2001. On Law Invariant Coherent Risk Measures, Adv. Math. Econ. 3, 8395.

Labuschagne, C.CA., Offwood, T.M., 2010. A note on the connection between the Esscher-Girsanov transform and the Wang trasnform. Insurance: Mathematics and Economics 47, 385-390.

Lansman, Z., 2010. On the Tail MeanVariance optimal portfolio selection. Insurance: Mathematics and Economics 46, 547-553.

Lewis, T., Thompson, J. W., 1981. Dispersive distributions and the connection between dispersivity and strong unimodality. J. Appl. Prob. 18, 76-90.

Müller, A., Stoyan, D., 2002. Comparison methods for stochastic models and risks.Wiley, New York.

Quiggin, J., 1982. A theory of anticipated utility. Journal of Economic Behavior and Organization 3, 323-343.

Rojo, J., He, G.Z., 1991. New properties and characterizations of the dispersive ordering. Statistics and Probability Letters 11, 365-372.

Saunders, I. W., Moran, P. A. P., 1978. On the quantiles of the gamma and F distributions. J. Appl. Prob. 15, 426-432.

Shaked, M., 1982. Dispersive ordering of distributions. J. Appl. Prob. $19,310-320$.

Shaked, M., Sordo, M.A., Suárez-Llorens, A. 2010. A class of locationindependent variability orders, with applications. Journal of Applied Probability 47, 407-425.

Shaked, M., Shanthikumar, J.G., 1998. Two variability orders. Probability in the Engineering and Informational Sciences 12, 1-23.

Shaked M., Shanthikumar J.G., 2007. Stochastic orders. Series: Springer Series in Statistics, Springer.

Schmeidler, D., 1989. Subjective Probability and Expected Utility without Additivity, Econometrica 57, 571-587.

Sordo, M.A., 2008. Characterizations of classes of risk measures by dispersive orders. Insurance: Mathematics and Economics 42, 10281034 
Sordo, M.A., 2009a. Comparing tail variability of risks by means of the excess wealth order. Insurance: Mathematics and Economics 45, 466-469.

Sordo, M.A., 2009b. On the relationship of location-independent riskier order to the usual stochastic order. Statistics and Probability Letters $79,155-157$.

Wang, S., 1995. Insurance pricing and increased limits ratemaking by proportional hazards transforms. Insurance: Mathematics and Economics 17, 43-54.

Wang, S., 1996. Premium calculation by transforming the layer premium density. ASTIN Bulletin 26, 71-92.

Wang, S., 2000. A Class of Distortion Operators for Pricing Financial and Insurance Risks, J. Risk Ins. 67, 15-36

Wang S, Young V.R., 1998. Ordering risks: Expected utility theory versus Yaari's dual theory of risk. Insurance: Math. and Econ. $22,145-161$

Yaari, M.E., 1987. The dual theory of choice under risk. Econometrica $55,95-115$ 